\title{
Etnografía escolar del celular como un recurso para la construcción de espacios de fuga ${ }^{1}$ \\ School ethnography of the cellphone as a resource for building scape spaces
}

Recibido: 18 de enero de 2017. Revisado: 3 de noviembre de 2017. Aceptado: 27 de noviembre de 2017.

\section{Daniela Hernández Silva ${ }^{2}$}

\begin{abstract}
Resumen
Este artículo socializa los resultados de una investigación etnográfica que abordó el uso del teléfono celular como un foco para analizar las dinámicas de socialización de los adolescentes en un colegio privado de la ciudad de Bogotá. Para ello se tuvieron en cuenta las interacciones dentro y fuera del aula de clases, abarcando tanto espacios regulados como informales. El análisis de dichos escenarios evidenció que el uso del móvil tiene un alto atractivo social para los adolescentes, dándole cabida aún en espacios donde está prohibido e inscribiendo su uso en lo que se denominó espacios de fuga.
\end{abstract}

\section{Palabras clave}

Socialización, escuela, regulación, celulares, espacios de fuga, adolescentes.

\begin{abstract}
This paper socializes the results of an ethnographic research that approach the cellphone use as a lens to analyze the teenager's socialization dynamics in a private School of Bogotá. To this end, interactions within and outside the classroom were considered, encompassing both regulated and informal spaces. The analysis of these scenarios showed that the use of the mobile phone has a high social attractiveness for adolescents, giving to it a place even in spaces where it is prohibited and inscribing its use in what is called here escape spaces.
\end{abstract}

\section{Keywords}

Socialization, school, regulation, cellphone, escape spaces, teenagers.

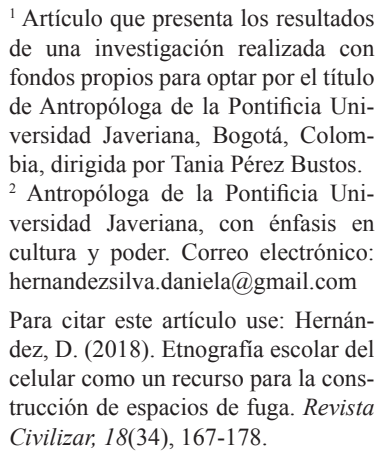

${ }^{1}$ Artículo que presenta los resultados de una investigación realizada con fondos propios para optar por el título de Antropóloga de la Pontificia Universidad Javeriana, Bogotá, Colombia, dirigida por Tania Pérez Bustos. 2 Antropóloga de la Pontificia Universidad Javeriana, con énfasis en cultura y poder. Correo electrónico: hernandezsilva.daniela@gmail.com

Para citar este artículo use: Hernández, D. (2018). Etnografía escolar del celular como un recurso para la construcción de espacios de fuga. Revista Civilizar, 18(34), 167-178. 


\section{Introducción}

Desde hace unas décadas el celular se ha venido posicionando crecientemente en la vida de sus usuarios, propagándose a gran velocidad alrededor del mundo. Inevitablemente estos instrumentos móviles se han inscrito en lo más esencial de la cotidianidad de millones de personas, transformándola $\mathrm{y}$, especialmente, modificando las dinámicas de socialización que antes se conocían. Con un profundo interés en comprender la transformación de dichas dinámicas, la investigación etnográfica que aquí se socializa pretendió analizar la inserción del celular en una Institución Educativa (IE) y su incidencia en las dinámicas escolares.

Así, este artículo presenta los resultados de un proceso de investigación comprendido entre los años 2014 y 2015 en la ciudad de Bogotá, exponiéndolos a través de tres momentos: el primero da cuenta de aspectos personales, metodológicos y teóricos claves en la construcción y el desarrollo de la investigación; el segundo, expone los hallazgos principales de la misma; y el tercero, presenta las conclusiones y las consideraciones finales a propósito de los hallazgos.

\section{Problema de investigación y método}

\section{Una ruptura como base.}

Mi rol como estudiante y docente me ha permitido testificar el privilegio que ha tenido en Colombia aquello que Sáenz (1997) llama pedagogía hacia la individualización, en la que se tiende a suprimir la expresión individual, que es vista como promoción del ocio y el desorden, dando prioridad al cumplimiento regulado del currículum formal encaminado a finalidades económicas y al orden social. Al mismo tiempo, ha puesto en evidencia el papel fundamental que tienen los dispositivos tecnológicos en el relacionamiento de jóvenes y adolescentes, cuyo uso está generalmente prohibido en las IE.
Siguiendo entonces el argumento de Berger y Luckman (1984), según el cual las características del mundo social de los estudiantes están distantes de las dinámicas que se ven obligados a seguir al interior del colegio, este trabajo se construyó sobre el reconocimiento de la ruptura entre las dinámicas escolares y las principales formas de socialización de los estudiantes.

\section{¿Qué, a quiénes y en dónde?}

Siendo el interés principal analizar las rupturas producidas en un ambiente pedagógico regulado, definí el salón de clases como el escenario primordial de observación debido a su carácter predominantemente formal (Díaz de Rada, 2005). Sin embargo, también incluí la vinculación de jóvenes por fuera del aula, con el propósito de abarcar de manera más completa el espectro de relaciones de los adolescentes.

Por su parte, prioricé un colegio privado ubicado en la ciudad de Bogotá ${ }^{1}$. Este es proveniente de una de las 29 comunidades católicas educadoras que llegaron a Colombia en el siglo XX. Fue fundado desde mediados de la década de 1960, contando con el apoyo de importantes comunidades religiosas y figuras políticas del momento, así como con la comunidad de Chapinero de Bogotá, localidad en la que desde entonces se encuentra ubicado. En la actualidad, la institución cuenta con gran reconocimiento académico y social en el país, es de carácter privado, y está conformado por 500 estudiantes aproximadamente, cuyas familias, por lo general, profesan la fe católica. Así, este lugar representó un sitio idóneo para hacer la investigación etnográfica, primero por haber sido construido sobre la base de las instituciones disciplinadas (Granes, 1998), y, segundo, gracias a mi relación preexistente con la institución, lo cual permitió que fueran abiertas las puertas para el desarrollo de la presente investigación.

Finalmente, el teléfono celular fue seleccionado como objeto principal de observación. 
Ello teniendo en cuenta que el móvil se ha convertido en un medio de comunicación de uso masivo e indispensable para la mayoría de personas (Odriozola, Labrador y Becoña, 2009), además del hecho de que es el dispositivo tecnológico al que más acceso tienen los jóvenes y adolescentes en Colombia (Páez, 2006).

Así, esta investigación pretendió analizar la forma en que se inserta el uso de celulares en las dinámicas reguladas que suceden al interior del aula de clases y cómo ello es configurado por la socialización entre pares. Por ende, el teléfono móvil devino en un lente de observación, es decir, en un lugar que materializaría dichas dinámicas y las haría visibles, a través del abordaje de la siguiente pregunta: ¿De qué manera se inserta el uso de celulares en el aula y cómo esto configura las dinámicas de socialización entre pares?

\section{¿Cómo?}

Para el levantamiento de la información se utilizaron principalmente cuatro herramientas metodológicas: la observación, un cuestionario, las conversaciones y las entrevistas no directivas.

\section{a) Observación.}

Esta herramienta fue utilizada durante el segundo semestre del año 2014 a los estudiantes de $8^{\circ}$, curso compuesto por 26 niños y 12 niñas. Se realizó lo que Coulon (1995) denomina observación directa, en la que el investigador hace parte de las actividades y mantiene contacto prolongado con un grupo, dando acceso a las prácticas cotidianas a través de las que la socialización acontece. Se efectuó de dos maneras: por una parte, a través de la observación prolongada en el aula, que consiste en la estancia en el aula durante un período de tiempo significativo, utilizando un diario de campo como instrumento para plasmar lo observado; esta es, según Lahire (2005), la herramienta metodológica más apropiada para hacer estudios etnográficos en el aula, pues permite identificar y analizar las formas de socialización en la escuela, al tiempo que le permite a la comunidad acostumbrarse a la presencia del investigador.

Por otra parte, a través de la observación participante, en la que se observó sistemática y controladamente mientras se tomaba parte en las actividades que se desarrollaban (Guber, 2001); fue clave para acceder a acciones espontáneas y no reguladas especialmente fuera de ella. Todo esto se aplicó teniendo en cuenta el concepto de currículo oculto, es decir, aquello que se enseña implícita y no explícitamente (Wilcox, 2005), que corresponde a los aprendizajes que no están expuestos como tales, pero que desempeñan un papel trascendental en la formación del sujeto social (Franzé y Jociles, 2008).

\section{b) Cuestionario.}

Durante el mismo periodo de tiempo y al mismo curso, les fue aplicado un cuestionario escrito, el cual representa una de las principales formas de recolección de información cuantitativa (Briones, 2002). Estuvo constituido por cinco preguntas de selección múltiple y cinco de respuesta abierta, y fue realizado con el propósito de brindar otro espacio de enunciación a los estudiantes y así, tener una fuente de información diferente para contrastar y analizar las coherencias e incoherencias de los datos extraídos desde las herramientas metodológicas cualitativas.

\section{c) Conversaciones.}

Las conversaciones fueron las charlas o diálogos que se dieron con estudiantes y profesores, caracterizadas por ser espontáneas, sin lineamientos y sin obligatoriedad. Permitieron un acercamiento casual y de contenido no formal. Supusieron la puesta en marcha de estrategias de acercamiento y de generación de lazos de confianza en los espacios que Bernete (2009) denomina "tiempo libre", donde se habla con más libertad y hay espacio para la conquista y el juego. 


\section{d) Entrevistas no directivas.}

Estas representan aquella relación social donde se realizan y se focalizan preguntas según un tema de interés (Guber, 2001). Esta herramienta, además de recolectar en palabras información que posteriormente sería transcrita, permitió visibilizar la perspectiva del informante más que solo la del investigador. Se entrevistó a una muestra arbitraria de cinco estudiantes de $8^{\circ}$, al profesor de la clase y a la psicóloga del colegio.

\section{e) Análisis de los datos.}

Este proceso consistió básicamente en cuatro etapas: la primera fue de categorización de la información recolectada según el escenario del que provenía (por fuera y al interior del aula de clases); en la segunda se realizó lo que Boyle (2003) llama análisis de contenido latente, es decir, ir más allá de lo explícito y procurar inferir el significado; la tercera etapa consistió en la identificación y construcción de viñetas etnográficas para ejemplificar las inferencias realizadas centrándome en momentos clave (Wilcox, 2005); y la cuarta consistió en construir un entramado de relaciones entre los datos que componían las viñetas etnográficas, conformando con ello el corpus interpretado que compuso el análisis e hizo posible la deducción de conclusiones.

\section{Etnografía, interaccionismo simbólico y socialización}

Esta investigación responde a un ejercicio etnográfico que pretendió observar, participar, describir, describir significativamente e interpretar (Jonorio, Velazco, García y Díaz de Rada, 2005). Produjo también nuevo conocimiento que ayuda a la comprensión del mundo social (Rockwell, 2005; Horst, Hjorth y Tacchi, 2012). Al reconocer la diversidad que cobija a los trabajos etnográficos, esta investigación se ubica simultáneamente dentro de las etnografías escolares y dentro de los estudios etnográficos de los medios de comunicación: pertenece a las primeras en tanto los estudiantes son sus sujetos-objetos de estudio (Jonorio et al., 2005) y el colegio su principal escenario (Axpe, 2003); y corresponde a los segundos, pues a través de una mirada cualitativa pretende comprender la vida cotidiana, el significado y las prácticas que hay a través de un dispositivo digital (Horst et al., 2012).

Así mismo, esta investigación tuvo como enfoque el interaccionismo simbólico, pues se realizó una observación continua y cuidadosa de las interacciones (Vásquez y Martínez, 1996), con el fin de comprender patrones de interacción a través del lenguaje y las actitudes (Axpe, 2003). Coincidiendo así con los postulados de este enfoque, que plantean que detrás de las formas en las que los individuos interactúan hay siempre un sentido latente que contribuye a la construcción de las acciones cotidianas (Coulon, 1995).

Finalmente, esta investigación realiza un aporte a los estudios de la socialización, trabajando este término sobre una base elaborada a partir de cuatro postulados: se tomó la noción de Lahire (2005), según la cual es un proceso de conformación del sujeto social; el argumento de Berthelot (1998), quien la refiere con los procesos de adquisición de conocimientos que permiten la interacción social; la definición de Parish (2001), quien la reconoce como la forma de llegar a hacer parte de una sociedad determinada; y finalmente, la perspectiva de Vásquez y Martínez (1996), donde se concibió como un proceso cotidiano que se da para la construcción de lazos sociales.

\section{Presentación de los resultados de investigación}

Los resultados y reflexiones a propósito del celular, su lugar de visibilidad y su incidencia en la socialización serán expuestos a través de (1) una aproximación al uso del móvil por fuera del aula y (2) del análisis de su uso al interior del salón de clases. 


\section{Por fuera del aula: espacios de evi- dencia espontánea.}

Los espacios de evidencia espontánea permitieron explorar una dimensión de la socialización de los estudiantes que no tuviera un carácter formal y obligatorio. Para exponer estos los hallazgos, utilizaré dos categorías: 1) ¿qué hace el sujeto a través de la pantalla? y 2) ¿cómo se relaciona el sujeto con la pantalla?

\section{a) ¿Qué hace el sujeto a través del móvil?}

Si tomamos estudios como el realizado por Ling (1997), quien concibe el uso del móvil como una acción sobre un dispositivo que requiere de lógicas básicas con una dimensión relacional, o por Campbell y Jin (2008), quienes lo refieren como un acto individualizado, a través del que hay expresión simbólica, podemos ver cómo la cuestión del uso del móvil ha sido concebida como un asunto que responde al qué hace el sujeto a través de la pantalla. Abordar dicha pregunta en esta investigación permitió la emergencia de dos asuntos.

\section{Celular como soporte y enlace de la vida social.}

Conversación informal con Andrés ${ }^{2}$, estudiante de $8^{\circ}$ :

Yo: ¿Para qué usas el celular?

Andrés: En cálculo lo uso para la calculadora, y bueno, como soy nuevo aquí, también lo uso para hablar con amigos de mi anterior colegio (Nota extraída del diario de campo de la autora, 22 de agosto de 2014).

Conversación con Lina, estudiante de 15 años a quien sus padres me habían pedido cuidar: "Lina: ¿Me acompañas a dónde Catalina? Ella vive aquí al lado y me gustaría verla" (Nota extraída del diario de campo de la autora, 22 de agosto de 2014).
Catalina era otra adolescente a quien habíamos conocido hacía tres años en un evento. Lina insistió, argumentando que eran amigas muy cercanas y que desde que se conocieron no se habían vuelto a ver. Como a Lina poco la dejaban salir, el celular había hecho posible la construcción de su amistad, así que las acompañé al encuentro. En él, la conversación fue tímida y débil, ambas mirando sus celulares $\mathrm{y}$ encontrando fluidez al comentar las cosas que veían en sus redes sociales. Justo antes de terminar la visita, Catalina tuvo la iniciativa de tomar una selfie con el objetivo de enviarla a una amiga mutua de ellas (escena construida a partir del diario de campo de la autora, 3 de octubre de 2014)

En las dos situaciones es visible cómo reconocen y utilizan el celular para darle continuidad a relaciones anteriores que querían mantener, o para construir relaciones que no son preexistentes. Si tomamos en cuenta que la movilidad en espacios físicos para los adolescentes es limitada por factores como la dependencia a los padres y la falta de ingresos económicos propios (Bernete, 2009), las redes utilizadas a través del celular devienen una parte fundamental del desarrollo de su vida social (Almansa, Fonseca y Castillo, 2013), respondiendo con ello a la necesidad social que tienen de establecer relaciones, de hacer parte de grupos paritarios (Arango, Bringué y Sábada, 2010; Páez, 2006), y de hacer visibles sus relaciones enlazándolas con distintos núcleos sociales. Por ende, el celular resulta ser un dispositivo con un alto atractivo social para los adolescentes (Almansa et al., 2013), ya que a través de él se hace posible la comunicación, construcción y exposición de relaciones sociales, soportando y enlazando vínculos por medio de su uso, favoreciendo así sus procesos de socialización.

\section{Vínculos emocionales con el celular.}

Después de estar un largo rato junto a Lina en silencio -pues como de costumbre ella 
estuvo escribiendo en su celular sin pronunciar palabra- ella alzó su mirada y me pidió que me acercara. Al hacerlo, ella reaccionó inmediata, natural e inconscientemente acercando su celular contra su pecho, con la clara intención de que yo no pudiera ver lo que estaba en su pantalla (Escena construida a partir del diario de campo de la autora, 3 de octubre de 2014).

Esa acción sugiere un deseo de privacidad, encontrando en el celular un lugar para experimentarla. Como lo menciona Van Weezel y Benavides (2009), una de las principales características del móvil para los adolescentes es que al usarlo pueden escapar del control de los padres, en tanto se puede conversar privadamente por ese medio, incentivando la autonomía, parte importante del desarrollo de su vida social (Bernete, 2009).

Por otra parte, Alejandra, una estudiante del colegio objeto de esta etnografía, comentó:

- "Facebook no lo uso para hablar con mi familia. O sea, a mí tampoco es que me guste mucho hablar por ahí, pero cuando lo hago es para hablar con mis amigos" (Entrevista no directiva a estudiante de $8^{\circ}, 24$ de septiembre de 2014).

Al igual que Alejandra, el 91\% de sus compañeros afirmaron, por medio del cuestionario aplicado, que las redes que usan a través del celular son para hablar con amigos antes que con familiares. Esto sugiere que ellos reconocen y quieren que sea reconocida cierta independencia por medio del uso que le dan al celular.

Este asunto ilustra cómo en el uso del móvil está adscrito un vínculo para enlazar afectivamente a los usuarios con el dispositivo. El trabajo de campo evidenció que dicho vínculo puede estar compuesto por asuntos que involucran las emociones de los adolescentes al permitir el reconocimiento externo de ciertas aptitudes que les permiten agradar y posicionarse socialmente.

\section{b) ¿Cómo se relaciona el sujeto con el móvil?}

Mientras hablaba con Andrés, noté que él no había soltado ni un segundo su celular de sus manos y que a pesar de que no lo había encendido ni una vez, había estado jugando con él entre sus dedos, cambiándolo de mano repetidamente y fijando su mirada en él. Me di cuenta que esa manía era una forma de uso del celular, pero ¿dónde encajaba en la literatura revisada? y ¿constituía un patrón de acción recurrente o había sido sólo un momento esporádico? Recordé entonces el encuentro entre Lina y Catalina, en donde pude notar que sin importar lo que hiciéramos ellas no soltaron el celular de sus manos ni un instante, y en vez de guardarlo, lo expusieron constantemente.

Me di cuenta entonces que estábamos frente a una forma poco explorada de lo que Abeele (2014) denomina mobile lifestyles, es decir, frente a un conjunto de prácticas y entendimientos que hay en torno al uso de los móviles y que esa manía de jugar, sostener y exponer el celular mientras se comparten espacios sociales, representa un patrón de acción a través del cual el comportamiento se materializa. Dicho patrón permitió pensar que lo que se hacía a través de la pantalla no era la única forma de abordar el uso del móvil, sino que también era posible pensar en que había formas particulares de relacionarse con ella. Ello abrió paso a la emergencia de otro asunto: el uso habitual del celular.

\section{Uso habitual del celular.}

Un uso habitual es una acción que se realiza continua y cotidianamente. Así mismo, refiere a un hábito cuya repetición puede producir falta de consciencia sobre las disposiciones físicas que componen la acción. Esta idea ha sido evidenciada por autores como Goggin (2012), quien habla del contacto perpetuo, implicando la comunicación constante; Carbonell, Fuster, Chamarro y Lusar (2012), quienes toman las consultas frecuentes al celular como un indica- 
dor de riesgo; Odriozola et al. (2009), quienes caracterizan el uso del móvil a través de la atención y dedicación de importantes periodos de tiempo diario. Estos trabajos corroboran el uso habitual del móvil, pero ¿qué hay detrás de que el celular sea utilizado habitualmente?

En primer lugar, el uso habitual del móvil se anida dentro de la construcción de relaciones sociales que son, en esencia, una práctica continua. Es decir, que si la construcción y perpetuación de relaciones sociales es una práctica cotidiana fundamental para el desarrollo social de los adolescentes (Arango et al., 2010; Páez, 2006) y el celular representa un dispositivo que lo hace posible, el uso del móvil resultaría ser una práctica regular y rutinaria, como bien lo expresa Alejandra:

"Bueno, pues yo tengo un Samsung en el que tengo internet todo el tiempo. La verdad lo uso todos los días y entre clases también" (Entrevista a estudiante de $8^{\circ}, 24$ de septiembre de 2014).

Algunos autores atribuyen este uso constante al FOMO (Fear of Missing Out), es decir, a la preocupación de quedarse por fuera de los círculos sociales (Carbonell et al., 2012). Así, detrás del uso habitual yacería una razón principalmente social. Por ende, los patrones de acción en torno a su uso se caracterizan por la exhibición continua, acompañada del constante contacto físico con el dispositivo móvil.

\section{Volviendo la mirada al Colegio}

En este apartado daré cuenta de cómo los hallazgos presentados en la sección anterior también configuran el escenario escolar, teniendo en cuenta que el aula está atravesada por dinámicas particulares de regulación y formalidad, como lo expresan algunos estudiantes:

Yo: ¿Les dejan usar celulares en el Colegio? Andrés: Pues hay unas clases en las que nos dejan usarlo, por ejemplo, en español para las lecturas. Claro que hay unas clases en las que los decomisan. Mejor dicho, hay profesores que molestan más que otros (Nota extraída del diario de campo de la autora, 22 de agosto de 2014).

Yo: ¿Cómo describirías el uso del celular dentro del Colegio?

Juan: Nos lo prohíben totalmente, pero la verdad es que si uno lo usa es porque uno ve que lo puede sacar (...)" (Entrevista a estudiante de $8^{\circ}, 24$ de septiembre de 2014).

Estas citas nos brindan una breve descripción del hecho de que el uso del celular dentro del escenario escolar es percibido como un elemento atravesado por dinámicas de prohibición. Expresan entonces una tensión entre la norma que es representada a través del decomiso y la interpretación relativa de dicha norma que configura su cumplimiento.

\section{La norma en el Colegio.}

El decomiso es una acción permitida y soportada por las normas escritas de la mayoría de instituciones escolares (Sarbach, 8 de julio de 2012). En el colegio priorizado para la presente investigación, el uso del móvil es calificado como una falta grave, es decir, como "un comportamiento que atenta contra los principios institucionales, perturbando el normal desarrollo de las actividades y -que- afecta gravemente las normas disciplinarias" (Manual de Convivencia, 2014, p. 125). Ante ello la psicóloga mencionó:

"Ellos pueden traer sus móviles, porque generalmente son los móviles. No los pueden usar en clase, aunque se sabe que ellos lo usan por debajo de cuerda" (Entrevista a psicóloga del Colegio: 15 de octubre de 2014).

Este comentario deja entrever que la forma en la que se cumple o no con la norma repercute en la interiorización de prácticas, lo que nos lleva a pensar que la normativa también traza lineamientos que configuran las interacciones en la escuela. 


\section{Reinterpretación de la norma según el escenario.}

El profesor de $8^{\circ}$, Francisco, en una de sus clases afirmó: "A pesar de las reglas, hay realidades que nos rodean y no las podemos cambiar. Por eso, hay momentos en que es mejor permitir el uso de celulares" (Nota extraída del diario de campo de la autora, 22 de agosto de 2014).

El comentario del profesor corrobora lo que Rockwell (2005) argumentó con respecto a que la norma educativa oficial en vez de incorporarse en las dinámicas de la escuela conforme a su formulación explícita es recibida y reinterpretada desde donde encuentra un lugar de agenciamiento. Desde la perspectiva de un estudiante, dicho postulado se entiende así:

Yo: ¿Es decir que usas el celular en clase aun sabiendo que no está permitido?

Alejandra: $\mathrm{Si}$

Yo: Y, ¿cómo sabes cuándo hacerlo?

Alejandra: Pues es que uno tiene cuidado de no sacarlo en clases con ciertos profesores que uno sabe que tienen más cuidado con eso y que se dan cuenta rápido.

Entrevista a estudiante de $8^{\circ}, 24$ de septiembre de 2014.

Es visible que no es cuestión de que haya prácticas que hagan parte de la norma y otras que no, sino que toda experiencia en el escenario escolar conforma las dinámicas en torno a la norma. Así, el comportamiento habitual dentro de la institución estaría basado en la resignificación de la norma, para lo que los márgenes de transgresión juegan un papel fundamental. Estos son, maneras socialmente aceptables de reinterpretar la norma (Vásquez y Martínez, 1996), e indican el momento, el lugar y las circunstancias específicas para que dicha norma sea configurada. En el caso de Alejandra, el margen de transgresión se delimitaría por las características del profesor con que esté.

\section{La clase del profesor Francisco: el ciclo de interacción en torno a la re- gulación.}

¿De qué manera dentro del entramado de regulación del aula tiene cabida el uso del celular? Para abordar esta cuestión, la estancia prolongada en el aula me permitió evidenciar una tendencia de comportamientos que constituían las dinámicas de interacción y que serán entendidos aquí como el ciclo de interacción en torno a la regulación:

-Antes de que el profesor llegara al salón, había libertad en el hacer, muchos estaban de pie y el volumen era elevado. Pero cuando ingresó al aula, todos se ubicaron de pie frente a sus puestos, respondieron a su saludo y después de dada la orden, se sentaron en silencio. El profesor informó que leerían y que podían hacerlo desde sus celulares. En ese instante el ambiente se intranquilizó, acompañado de susurros en creschendo y de sonidos de cremalleras y puestos que se abrían y cerraban. En esos 2 minutos el profesor no manifestó intención de calmarlo, como si hubiera dado permiso de dispersión. Después, todo volvió a la calma de forma natural.

Aunque tenían la autorización de usar el celular, la mayoría lo usaba a escondidas, poniéndolo en sus piernas o cubriéndolo con sus manos. Por su parte, el profesor estaba atento, caminando en la tarima del salón y mirando fijamente a los estudiantes, como haciéndoles saber que estaba pendiente de lo que hacían y que tenía el control, produciendo completo silencio en el salón. Al cabo de un par de minutos, el profesor se sentó y comenzó a leer algo en su escritorio. Entonces, el espacio se dispersó, se escucharon susurros de la voz y de las cosas que materializaban la desconcentración de los niños. De repente, el profesor se puso de pie y al ver que un estudiante utilizaba Facebook le decomisó su celular. Con ello recobró la atención y el respeto de su clase, pues instantáneamente hicieron silencio y muchos guardaron sus móviles. De inmediato, el profesor se hizo en la parte trasera del salón para observar las pantallas de los celulares en uso, acción que, después de 5 minutos, se fue 
desvaneciendo con el traslado del profesor a la parte frontal del salón, causando con ello que el volumen del salón aumentara hasta que el ambiente estuvo disperso nuevamente (Escena construida a partir del diario de campo de la autora, 3 de septiembre de 2014).

$\mathrm{Si}$ los elementos que componen el ciclo de interacción en torno a la regulación son desglosados y analizados se podrían asemejar al juego con una cometa, donde para "soltar la pita" es necesario "atraerla". Es como si antes de la llegada del profesor estuvieran "libres", y el profesor atrajera "la pita" para comenzar, formando un espacio muy regulado y generando tensión que se corta con la instrucción de usar el celular. Ello hace que se suelte la "pita" y les sea permitido un espacio en el que se resuelva la tensión. Después viene un momento en que fluye naturalmente, hasta que se pierde toda regulación, pues los estudiantes de dispersan y utilizan el celular para fines no académicos, instante en el que se atrae la "pita" por medio de un mecanismo de control -el decomiso- que regula el espacio. Finalmente, la suelta poco a poco mediante el traslado físico a un lugar no vigilante en el que no ejerció ningún mecanismo de control, llevando a la resolución de la tensión.

Aparece así la tensión y la resolución de dicha tensión como una característica anclada a las dinámicas del aula. La tensión sería un resultado de la regulación del uso del celular al aplicar un mecanismo de control, mientras que la resolución caracteriza a los espacios en los que se rompe con la instrucción. Lo cierto es que la tensión siempre termina encontrando una salida para ser resuelta, salida que aquí llamaremos espacios de fuga.

Los espacios de fuga son entonces aquellos momentos de escape a la tensión que produce la regulación en el aula, es decir, momentos en los que se rompe con la instrucción, el orden o las dinámicas precedentes. Eso implica que la mayoría de veces, se hagan efectivos en momentos en que se transgrede una instrucción, para lo cual es necesario que se construyan for- mas socialmente aceptadas de hacerlo. Por ello, los estudiantes adoptan y construyen estrategias que les posibilita tenerlos en ambientes muy regulados. Muchos de los estudiantes afirmaron utilizar el celular escondido detrás o debajo de algo, y otros mientras el profesor está lejos o distraído. Así se crean, comparten y ponen en marcha una serie de disposiciones corporales que permiten usar -y esconder- el móvil, haciendo de los espacios de fuga en el aula espacios característicamente creativos.

\section{Conclusiones}

A través de esta investigación el concepto de socialización fue transversalmente abordado y continuamente construido, evidenciando los siguientes aspectos:

Se reconoció que en los procesos de socialización de los adolescentes la construcción de relaciones sociales es una necesidad social fundamental (Vásquez y Martínez, 1996), así que el uso del móvil supliría dicha necesidad a través de la creación y perpetuación de lazos sociales.

Se identificó una dimensión emocional de la socialización, evidenciando que el reconocimiento de aquellos asuntos emocionales es indispensable para la construcción y el posicionamiento social, ya que les permite a los adolescentes hacer parte de una sociedad determinada (Parish, 2001).

Se reconoció el carácter rutinario y constante de la socialización, implicando que ésta sea desarrollada en la cotidianidad a través de prácticas continuas y constantes, que deben ser aprendidas y que hacen posible la interacción social (Berthelot, 1998).

Teniendo lo anterior presente y sin ánimo de ofrecer una definición cerrada ni concluyente, sino proponiendo una noción contextualizada en esta investigación, el concepto de socialización enfocado en los adolescentes a través del uso 
de celulares hace alusión aquí a aquellos procesos que aportan a la conformación social del adolescente, que responden en gran medida a la necesidad de la construcción de relaciones sociales para las cuales el reconocimiento emocional se hace imprescindible, implicando una acción habitual que se materializa en las disposiciones corporales que tienen con respecto a aquellos objetos que los rodean.

Ahora bien, para dar cuenta de cómo se inserta el uso del celular en el aula, fue presentada una serie de complejas relaciones e interacciones a través de las cuales se concluyó que dentro del colegio el uso del teléfono celular se anida en los espacios de fuga. Pero entonces, ¿qué hay detrás de que el teléfono móvil sea seleccionado como esa forma de fuga por excelencia?, ante lo cual el análisis de los datos sugiere que detrás de él hay un objeto deseado, socialmente atractivo, cuyo uso tiene implicaciones efectivas y deseadas para la socialización de sus usuarios.

Así, las implicaciones que hay detrás del uso del celular por parte de los adolescentes estarían fuertemente ligadas a la socialización. $\mathrm{Y}$ a pesar de ser las mismas tanto dentro como fuera del aula, se diferencian en tanto que los lugares desde los cuales se hacen visibles son distintos, pues la exposición del móvil oscila constantemente entre el orgullo y el escondite según el escenario en el que se use.

Vale la pena aclarar que los datos presentados no sugieren que la norma tenga que ser reforzada en las instituciones educativas en vista de que es frecuentemente transgredida. Por el contrario, se pretende demostrar que los espacios de fuga son parte del ciclo de interacción en torno a la regulación, y por eso más normatividad no los agotaría.

Teniendo en cuenta además el atractivo y la función social del celular, considero que los datos obtenidos aquí no deben conducir a la extinción del uso del móvil en los colegios, pues ello sería coartar a los adolescentes de una parte importante de su desarrollo social. Por ende, sugiero que los colegios acepten la pertenencia regulada de estos dispositivos dentro de las instituciones y maximicen su uso a través de un trabajo de concienciación del uso adecuado de estos dispositivos para propagar su utilidad académica y social. Con ello se pretendería lograr una conciliación entre la normativa del colegio, la socialización fundamental de sus estudiantes adolescentes y las formas de su uso.

\section{Notas}

1 El nombre del colegio permanecerá anónimo en el marco del presente artículo por motivo de protección de la identidad a solicitud de los funcionarios del mismo.

2 De conformidad con el Decreto 1673 de 1994 en el Artículo 1 de la Constitución Colombiana, todos los nombres que hagan alusión a informantes menores de edad en el presente artículo serán cambiados para proteger su identidad.

\section{Referencias}

Abeele, V. (2014). Mobile lifestyles: Conceptualizing heterogeneity in mobile youth culture. New Media y Society, 1 (9), 1-19. Recuperado de http://nms.sagepub.com/content/ early/2014/09/16/1461444814551349.full

Almansa, A., Fonseca, O. y Castillo, A. (2013). Redes sociales y jóvenes. Uso de Facebook en la juventud colombiana y española. Comunicar: Revista científica iberoamericana de comunicación $y$ educación, 40, 127- 134.

Arango, G., Bringué, X. y Sádaba, C. (2010). La generación interactiva en Colombia: adolescentes frente a la Internet, el celular y los videojuegos. Anagramas: Rumbos y sentidos de la comunicación, 9 (17), 45-56. 
Axpe, M. A. (2003). La investigación etnográfica en el campo de la educación. Una aproximación meta-analitica. San Cristóbal de la Laguna: Universidad de la Laguna.

Berger, P. L. y Luckman, T. (1984). La construcción social de la realidad. Buenos Aires: Amorrortu editores.

Bernete, F. (2009). Usos de las TIC, Relaciones sociales y cambios en la socialización de las y los jóvenes. Revista de estudios de juventud, 10(88), 97-114. Recuperado de http://www.injuve.es/sites/default/files/ RJ88-08.pdf

Berthelot, J.M. (1998). Reflexions sur la pertinence du concept de socialization, en Analyses des modes de socialization. Lyon : Université Louis Lumière Lyon II.

Boyle, J. (2003). Estilos de etnografía. En J. Morce (Ed.), Asuntos críticos en los métodos de investigación cualitativa (pp.185216). Medellín: Editorial Universidad de Antioquia.

Briones, G. (2002). Metodología de la investigación cuantitativa en las ciencias sociales. Bogotá: ARFO Editores e impresores Ltda.

Campbell, S. y Jin, Y. (2008). Social Implications of Mobile Telephony: The Rise of Personal Communication Society. Sociology Compass, 2 (2), 371-387.

Carbonell, X., Fuster, H., Chamarro, A. y Lusar, U. (2012). Adicción a Internet y móvil. Una revisión de estudios empíricos españoles. Papeles del psicólogo, 33(2), 82-89.

Coulon, A. (1995). Etnometodología y educación. Barcelona: Editorial Paidós.

Díaz de Rada, A. (2005). ¿Qué obstáculos encuentra la etnografía cuando se practica en las instituciones escolares? En: A. Franzé, A y M. I. Jociles (Eds.), ¿Es la escuela el problema? perspectivas socioantropológicas de etnografía y educación. Madrid: Trotta.

Franzé, A., y Jociles, M. I. (2008). ¿Es la escuela el problema? perspectivas socioantropológicas de etnografía y educación. Madrid: Trotta.

Goggin, G. (2012). The iPhone and communication. En L. Hjorth, J. Burguess e I. Richardson, Studying mobile media: Cultural technologies, mobile communication, and the iPhone. UK: Routledge.

Granes, C. (1998). Nuevas tecnologías, identidad y socialización [Microficha] (Tesis de pregrado). Pontificia Universidad Javeriana, Bogotá.

Guber, R. (2001). La etnografía método, campo y reflexividad (1a ed.). Bogotá: Norma.

Horst, H., Hjorth, L. y J. Tacchi (2012). Rethinking ethnography: An introduction. Media International Australia, 145, 86-93.

Jonorio, M., Velazco, F., García, J. y Díaz de Rada, A. (2005). Lecturas antropológicas para educadores. El ámbito de la antropología de la educación y de la etnografía escolar. Madrid: Trotta.

Lahire, B. (2005). Un sociólogo en el aula: objetos en juego y modalidades. En A. Franzé y M. I. Jociles, ¿Es la escuela el problema? perspectivas socioantropológicas de etnografía y educación (pp. 49-61). Madrid: Editorial Trotta.

Ling, R. (1997). One Can Talk About Common Manners! The Use of Mobile Telephones in Inappropriate Situations. En L. Haddon (Ed.), Themes in Mobile Telephony Final Report of The COST 248 Home and Work 
Group. Recuperado de http://richardling. com/papers/ 1997_One_can_talk_about_ common_manners.pdf

Odriozola, E., Labrador F. J. y Becoña E. (2009). Adicción a las nuevas tecnologías en adolescentes y jóvenes. Madrid: Pirámide.

Páez, A. E. (2006). La relación jóvenes -TIC. Una lectura cualitativa. Hologramática, 5(1), 41-54.

Parish, S. M. (2001). Socialización. En T. J. Barfield y C. M. Sánchez-Rodrigo (Eds.), Diccionario de antropología (pp. 587- 588). Barcelona: Edicions Bellaterra.

Rockwell, E. (2005). Del campo al texto: dilemas del trabajo etnográfico. En A. Franzé, A y M. I. Jociles (Eds.), ¿Es la escuela el problema? perspectivas socioantropológicas de etnografía y educación. Madrid: Editorial Trotta.
Sáenz, J. (1997). Hacia una pedagogía de la subjetivación. Revista Educación y Pedagogía, 9-10 (19-20), 113-135.

Sarbach, A. (8 de julio de 2012). Socialización, pactos e innovación educativa [carbonilla. net]. Recuperado de: http://carbonilla. net/2012/07/08/socializacion-escolar/

Van Weezel, A. y Benavides, C. (2009). Uso de teléfonos móviles por los jóvenes. Cuadernos de información, 25, 5-14.

Vásquez, A. y Martínez, I. (1996). La socialización en la escuela. Una perspectiva etnográfica (1a ed). Barcelona: Paidós.

Wilcox, K. (2005). La etnografía como una metodología y su aplicación al estudio de la escuela: una revisión. En H. Velazco, J. García y A. Díaz de Rada (Eds.), Lecturas antropológicas para educadores. El ámbito de la antropología de la educación $y$ de la etnografia escolar (pp. 95-127) Madrid: Trotta. 